LES CONDITIONS ASSOCIÉES À UNE BONNE ADAPTATION AU RÔLE PATERNEL

\title{
QUELLES SONT LES CONDITIONS ASSOCIÉES À UNE BONNE ADAPTATION AU RÔLE PATERNEL POST-RUPTURE?: PARCOURS PATERNELS ET POINTS DE VUE DE PÈRES
}

\author{
JUDITH GAUDET \\ Université du Québec à Montréal \\ et \\ ANNIE DEVAULT \\ Université du Québec en Outaouais
}

\begin{abstract}
RÉSUMÉ
Cet article présente les résultats d'un volet d'une recherche qualitative qui vise à identifier les différentes conditions (psychologiques et sociales) qui semblent favoriser ou nuire à l'adaptation des pères à leur rôle parental après une rupture conjugale. Les analyses de récits de vie de 27 pères séparés ou divorcés qui sont présents dans la vie de leurs enfants ont permis d'identifier 4 trajectoires paternelles adaptatives postrupture. Ces trajectoires ou parcours de vie renvoient aux événements, obstacles, stratégies et facilitateurs qui caractérisent leur expérience post-rupture. Les éléments qui influencent positivement l'adaptation à leur rôle parental varient selon les trajectoires. Toutefois, certains sont communs à l'ensemble des pères. Ils concernent leur capacité à réaliser des compromis et des sacrifices importants avec l'ex-conjointe relativement à leur entente de séparation ou de divorce, leur croyance en l'importance de leur rôle dans la vie de leurs enfants, leur capacité à accepter ou à demander de l'aide après la rupture et leurs démarches proactives pour accéder à des conditions satisfaisantes d'exercice de leur rôle parental.
\end{abstract}

L'Enquête longitudinale nationale sur les enfants et les jeunes (ELNEJ) indique que près de $25 \%$ des enfants canadiens nés en 1987-88 ont connu la séparation ou le divorce de leurs parents avant l'âge de 6 ans (Marcil-Gratton \& Le Bourdais, 1999). Cette étude montre aussi que le lien entre le père et l'enfant tend à se fragiliser après une rupture. Parmi les enfants qui demeurent chez leur mère 5 ans après la séparation ou le divorce $(87,3 \%$ des enfants), $17,8 \%$ d'entre eux voient leur père à toutes les 2 semaines, $12,9 \%$ les voient une fois par semaine, $32,2 \%$ voient leur père de temps à autre et $24,2 \%$ ne les voient plus. En ce qui concerne les autres enfants, $7,2 \%$ d'entre eux demeurent chez leur père et $5,5 \%$ habitent à la fois chez leur mère et leur père selon un horaire partagé.

Considérant ces résultats, les chercheurs se sont attelés à mieux comprendre ce qui pourrait expliquer et prévenir le désengagement paternel. La plupart des recherches ont porté sur les variables et trajectoires parentales associées au désengagement graduel des

La rédaction de cet article de thèse de doctorat a été possible grâce à une bourse obtenue par la première auteure du Fonds québécois pour la recherche sociale (FQRSC) et à une subvention de la Direction de la santé publique de l'Outaouais obtenue par la deuxième auteure. Nous tenons à remercier Camil Bouchard, Ph.D., et Nancy Guberman, Ph.D., pour leurs précieux commentaires et Maryse Dassylva pour la transcription des entrevues et la validation des analyses. 


\section{REVUE CANADIENNE DE SANTÉ MENTALE COMMUNAUTAIRE}

pères après une rupture ou à leur absence en se basant souvent sur des données recueillies auprès des mères (Gaudet, Devault, \& Bouchard, 2005). Un moins grand nombre de chercheurs ont poursuivi l'objectif de documenter l'expérience des pères séparés encore présents dans la vie de leurs enfants et d'identifier les facteurs qui, selon eux, ont nui mais, surtout, contribué à leur bonne adaptation parentale et au maintien de l'exercice de leur rôle parental. Pourtant, les facteurs qui favorisent une meilleure adaptation à une situation stressante (facteurs de protection) ne sont pas nécessairement l'inverse de ceux qui y nuise (facteurs de risque) (Rutter, 1987).

De plus, une meilleure connaissance de tels facteurs de protection pourrait contribuer à la mise en place de programmes de promotion plus efficaces et encore mieux adaptés à la réalité des pères séparés. D'ailleurs le besoin de ce type d'intervention représente l'une des recommandations du Comité de travail en matière de prévention et d'aide aux hommes (2004).

Au Canada, seulement trois études ont donné la parole à des pères séparés ou divorcés qui étaient encore présents dans la vie de leurs enfants dans le but d'identifier les facteurs associés au maintien de leur engagement parental (Allard, Bourret, Tremblay et coll., 2004; Kruk, 1991; Le Bourdais, Juby, \& Marcil-Gratton, 2001). L'une d'entre elles (Allard et al., 2004) a été réalisée auprès de pères québécois. Elle documente en profondeur la situation des pères séparés par l'entremise d'entretiens semi-dirigés et elle s'est réalisée auprès d'un petit échantillon de pères vivant en contexte de pauvreté $(n=15)$. Notre étude se situe en complémentarité avec les travaux de ces chercheurs.

La littérature scientifique montre que maintien de l'engagement paternel post-rupture est associé aux éléments suivants: (a) la diminution de l'intensité des conflits entre exconjoint(e)s après la rupture (Arditti \& Bickley, 1996; Minton \& Pasley, 1996) et la capacité des parents à communiquer ensemble au sujet de l'éducation des enfants (Rosenbaum, 2000); (b) le fait que le père ait de bons revenus et/ou un bon niveau d'éducation (Cooksey \& Craig, 1998; Le Bourdais et al., 2001; Ottosen, 2001); (c) la proximité physique du père de la résidence des enfants (Cooksey \& Craig 1998; Le Bourdais et al., 2001; Lewis, Maka, \& Papacosta, 1997; Stephens, 1996); (d) une forte identification du père à son rôle parental et la priorisation de son rôle de père par rapport à ses autres rôles (employé, ami, amoureux) (Allard et al., 2004; Ihinger-Tallman, Pasley, \& Buehler, 1995; Minton \& Pasley, 1996; Stone \& McKenry, 1998); (e) une bonne adaptation psychologique $\mathrm{du}$ père après la rupture conjugale (Hetherington \& Kelly, 2002; Kruk, 1991; Rettig, Leichtentritt, \& Stanton, 1999; Stone, 2001); (f) une relation père-enfant post-séparation harmonieuse ou jugée satisfaisante par le père (Hetherington \& Kelly, 2002; Le Bourdais et al., 2001; Stone \& McKenry, 1998); et (g) la capacité à demander de l'aide et le fait d'être soutenu par son entourage (Allard et al., 2004; Hethrington \& Kelly, 2002).

\section{CADRE THÉORIQUE}

Sur les plans théorique et conceptuel, cette étude exploratoire s'inspire entre autres de la conception écologique du développement humain de Bronfenbrenner (1979). L'approche écologique considère que le développement est déterminé par la qualité et la continuité des liens qui s'établissent entre l'individu (ontosystème) et ses environnements proximaux, divisés en microsystème (famille, ami[e]s et milieux de travail) et mésosystème (liens entre les microsystèmes); et par l'influence indirecte des environnements distaux, divisés en exosystème (institutions, conseils d'administration) et macrosystème (valeurs et croyances sociales). Cette approche s'avère pertinente, car l'examen de la littérature scientifique 
indique que tant des variables psychologiques que sociales jouent un rôle important dans la vie des pères séparés. De plus, peu de chercheurs dans le domaine ont considéré ces deux types de variables en interaction.

La perspective d'adaptation aux transitions familiales de Cloutier et ses collègues (1997) s'est également ajoutée au cadre théorique de base, étant donné que la recherche vise à documenter les facteurs qui sont favorables à une bonne adaptation au rôle paternel post-rupture. Selon ces auteurs, la clé de l'adaptation repose sur la continuité et le maintien des rôles malgré le changement. Plus spécifiquement, les parents s'adaptent plus aisément à une transition s'ils peuvent continuer à exercer leur rôle au sein de la nouvelle configuration familiale et y entretenir des liens de qualité. Leur adaptation est également facilitée s'ils sont en mesure de prévoir le changement et s'ils sentent qu'ils peuvent agir sur leurs nouvelles conditions de vie et conserver un pouvoir décisionnel. Enfin, les parents s'ajusteront mieux à une transition familiale s'ils sont en mesure de répondre à leurs besoins matériels et socio-affectifs et s'ils peuvent recevoir du soutien leur permettant de dédramatiser la transition qu'ils vivent.

\section{OBJECTIFS DE L'ÉTUDE}

Les objectifs d'un des volets de cette étude exploratoire consistent à (a) décrire et comprendre l'expérience de vie des pères qui sont toujours en contact avec leurs enfants après la rupture conjugale et (b) identifier les principaux facteurs (caractéristiques psychologiques et sociales des pères, de leur nouvelle configuration familiale et de leurs milieux de vie) qui selon eux ont facilité ou, au contraire, compliqué l'exercice de leur rôle parental.

\section{MÉTHODOLOGIE}

\section{Description de l'échantillon}

Des entrevues individuelles semi-dirigées ont été menées au cours des années 2001 et 2002 auprès de 27 pères $^{1}$ en provenance de la ville de Gatineau, qui étaient séparés depuis au moins 2 ans $^{2}$ et qui étaient toujours présents dans la vie de leurs enfants (voyaient leurs enfants au moins une fin de semaine sur deux). Le recrutement s'est réalisé par l'entremise d'annonces parues dans les journaux locaux et dans différents lieux publics: commerces et organismes communautaires. L'échantillon a été complété grâce à la méthode boule-deneige: les participants nous ont référé à d'autres pères séparés qu'ils connaissaient (Patton, 1987). Les entrevues duraient en moyenne $1 \frac{1}{2}$ heures et elles se déroulaient dans les bureaux de l'université. Huit pères répartis en deux groupes ont accepté de participer à un groupe de discussion et de validation des analyses préliminaires (tous les pères avaient été invités à participer à cette rencontre). Les deux rencontres de validation ont duré $2 \frac{1}{2}$ heures chacune. Toutes les entrevues ont été enregistrées sur bande-audio avec le consentement écrit des participants et elles ont été retranscrites. Tous les participants ont complété une fiche signalétique à la fin des entrevues.

Les participants étaient séparés depuis 5,8 ans en moyenne (cela variait entre 2 et 10 ans), et $80 \%$ d'entre eux avaient été mariés à la mère de leurs enfants. Au moment des entrevues, $40 \%$ des participants avaient une nouvelle conjointe. Tel que le montre le tableau 1, les participants disposaient de modalités de garde variées. 
REVUE CANADIENNE DE SANTÉ MENTALE COMMUNAUTAIRE

\section{TABLEAU 1}

Distribution de l'échantillon selon les modalités de garde $(N=27)$

\begin{tabular}{|c|c|}
\hline Modalités de garde & $\%$ \\
\hline $\begin{array}{c}\text { Garde physique partagée directement après la séparation (plus de 40\% du temps } \\
\text { passé avec les enfants) }\end{array}$ & 44 \\
\hline $\begin{array}{c}\text { Garde physique partagée après quelques années de « garde aux 15 jours » (entre 1 } \\
\text { et 5 ans après la séparation) }\end{array}$ & 19 \\
\hline Droits de visite aux 15 jours et pendant les congés scolaires & 37 \\
\hline
\end{tabular}

\section{TABLEAU 2}

Distribution de l'échantillon selon les revenus annuels bruts $(N=25)$

\begin{tabular}{|c|c|}
\hline Revenus annuels bruts & \% \\
\hline 50000 \$ et plus & 48 \\
\hline entre 40000 \$ et $49999 \$$ & 16 \\
\hline entre 30000 \$ et $39999 \$$ & 12 \\
\hline entre 20000 \$ et $29999 \$$ & 24 \\
\hline
\end{tabular}

\section{TABLEAU 3}

Distribution de l'échantillon selon le niveau de scolarité $(N=25)$

\begin{tabular}{|c|c|}
\hline Niveau de scolarité & $\mathbf{\%}$ \\
\hline Diplôme de $2^{\mathrm{e}}$ cycle universitaire & 8 \\
\hline Diplôme de $1^{\mathrm{er}}$ cycle universitaire ou certificat & 40 \\
\hline Diplôme du collégial (technique) & 36 \\
\hline Diplôme d'études professionnelles du secondaire & 16 \\
\hline
\end{tabular}

Les participants étaient âgés en moyenne de 40 ans. On comptait 1,3 enfants par père. Ils étaient âgés de 11 ans en moyenne. Les tableaux 2 et 3 indiquent que les participants provenaient de milieux socio-économiques variés.

Tous les pères rencontrés étaient caucasiens, francophones et d'origine canadienne, sauf un qui était d'origine mexicaine.

\section{Collecte et analyse des données}

Nous avons utilisé une méthodologie de recherche qualitative qui, pour la collecte de données, s'inspire de la théorisation ancrée de Glasser et Strauss (1967). Cette méthodologie implique un mode de travail itératif: l'analyse est concourante à la collecte de données. Cette façon de procéder permet de documenter de manière plus approfondie les facteurs qui, après analyse, semblent jouer un rôle important dans la compréhension du phénomène à l'étude, et de vérifier auprès des prochains participants si ces facteurs ont également joué un rôle important dans leur trajectoire de vie.

Ainsi, il s'est avéré pertinent de documenter de façon plus approfondie au fil des entrevues (a) l'histoire de la rupture conjugale (qui l'a initiée? comment a-t-elle été négociée?), (b) le processus de deuil amoureux, (c) la qualité relationnelle entre les ex-conjoint(e)s et leur niveau de collaboration concernant l'éducation des enfants (coparentalité) et (d) 


\section{LES CONDITIONS ASSOCIÉES À UNE BONNE ADAPTATION AU RÔLE PATERNEL}

l'intensité d'engagement paternel pré-rupture. De plus, après l'analyse des premières entrevues, il devenait incontournable de rencontrer des pères qui disposaient de modalités de garde physique diversifiées. Cette façon de procéder réfère à la notion d'échantillonnage théorique de Glasser et Strauss (1967): la documentation de contextes parentaux diversifiés et l'approfondissement graduel de certains thèmes qui se révèlent centraux en cours de route permettent d'enrichir et de nuancer l'analyse de données.

L'analyse de contenu des entrevues s'est déroulée en s'inspirant de l'approche de théorisation ancrée proposée par Paillé (1994) et du paradigme de codification axiale de Strauss et Corbin (1998). Les grandes étapes prescrites par ces auteurs ne se déroulent pas de façon linéaire et séquentielle, mais bien dans une logique itérative.

L'étape de codification. Cette étape consiste à résumer en de courtes phrases les éléments importants des entrevues en demeurant le plus près possible du sens premier du discours des participants (ex: "peur du père de perdre le contact avec ses enfants »). Toutes les entrevues ont été codifiées à l'aide du logiciel d'analyse qualitative N'Vivo. À cette étape, on réalise une analyse verticale des entrevues.

L'étape de catégorisation. Cette étape consiste à regrouper des codes se référant à un même thème dans le but de lui attribuer une étiquette qui renvoie à un phénomène plus large et qui présente une richesse conceptuelle plus grande ( $2^{\mathrm{e}}$ niveau conceptuel). Le tableau 4 présente les principales catégories qui ont émergé des analyses. Certaines sont plus spécifiques et elles sont développées selon des critères d'intensité (niveau d'harmonie entre les ex-conjoints: conflictuel, bon ou forcé) ou d'évolution dans le temps (historique des différentes modalités de garde depuis la séparation). D'autres sont plus globales et visent à rendre compte des différentes déclinaisons d'un thème central, comme la catégorie qui traite des difficultés rencontrées par les pères.

Une fois ces étapes complétées, une assistante de recherche a validé la catégorisation de sept entrevues sélectionnées au hasard. À partir des verbatims d'entrevues, elle devait valider les éléments identifiés par la chercheure et indiquer ceux qui avaient été omis. Ces éléments lui étaient présentés sous la forme de résumés d'entrevues organisés autour des principales catégories d'analyse. Tous les éléments ressortis par la chercheure l'ont également été par l'assistante.

L'étape de la mise en lien des catégories. Plus la catégorisation avançait, plus il devenait évident que des sous-groupes de pères se distinguaient les uns des autres en fonction de leur histoire de séparation, de leur processus de négociation de cette dernière et des modalités de garde et des difficultés rencontrées. Il est alors apparu important de procéder à un deuxième niveau d'analyse dans lequel on cherchait à identifier quelles combinaisons de catégories caractérisaient le mieux les différents sous-groupes de pères. On se demandait par exemple dans quels contextes on retrouvait davantage le même type de difficultés et de réactions face à celles-ci.

Afin d'atteindre cet objectif, nous avons d'abord croisé entre elles des variables sociodémographiques et/ou des catégories à l'aide du logiciel en vue de générer des matrices d'intersection. Celles-ci permettent d'observer le nombre de participants qui présentent les caractéristiques recherchées. Toutefois, ces croisements réalisés entre des catégories souvent statiques ne suffisaient pas à décrire l'histoire parentale de chacun dans une perspective davantage dynamique et chronologique. Afin d'y arriver, il a fallu retourner aux verbatims, cette fois en cherchant à identifier selon une logique chronologique les comportements du père de même que ceux de son environnement. Tout ce qui concerne la chronologie des événements a été noté par l'entremise de mémos analytiques (notes syn- 


\section{REVUE CANADIENNE DE SANTÉ MENTALE COMMUNAUTAIRE}

thèse de l'analyste qui sont associées à chaque entrevue et enregistrées dans le dossier d'analyse). Ces différentes étapes ont permis d'identifier quatre trajectoires parentales.

\section{TABLEAU 4}

Synthèse des principales catégories d'analyse

\begin{tabular}{|c|c|}
\hline 1. Type de séparation & $\begin{array}{l}\text { 1.1 Graduelle } \\
\text { 1.2 Choc } \\
\text { 1.3 Rapide mais prévue }\end{array}$ \\
\hline 2. Initiateur/trice de la séparation & $\begin{array}{l}\text { 2.1 Père } \\
\text { 2.2 Mère } \\
\text { 2.3 De commun accord }\end{array}$ \\
\hline $\begin{array}{l}\text { 3. Degré d'engagement paternel avant la } \\
\text { séparation }\end{array}$ & $\begin{array}{l}\text { 3.1 Élevé } \\
\text { 3.2 Moyen } \\
\text { 3.3 Faible }\end{array}$ \\
\hline $\begin{array}{l}\text { 4. Processus de négociation de la } \\
\text { séparation }\end{array}$ & $\begin{array}{l}\text { 4.1 Médiation } \\
\text { 4.2 Tribunal } \\
\text { 4.3 Amiable }\end{array}$ \\
\hline 5. Évolution des modalités de garde & $\begin{array}{l}\text { 5.1 Partagée dès le début } \\
\text { 5.2 Partagée après quelques années } \\
\text { 5.3 Droits de visite aux } 15 \text { jours depuis toujours }\end{array}$ \\
\hline $\begin{array}{l}\text { 6. Degré d'entente ente les ex- } \\
\text { conjoint(e)s }\end{array}$ & $\begin{array}{l}6.1 \text { Bon } \\
\text { 6.2 Forcé } \\
6.3 \text { Conflictuel } \\
\end{array}$ \\
\hline $\begin{array}{l}\text { 7. Facteurs perçus comme ayant facilité } \\
\text { l'adaptation au rôle paternel post- } \\
\text { rupture }\end{array}$ & $\begin{array}{l}\text { 7.1 Qualité de la relation entre les ex-conjoint(e)s: respect et } \\
\text { ouverture } \\
\text { 7.2 Comportement affectueux et dédié du père à l'égard de ses } \\
\text { enfants } \\
\text { 7.3 Comportement affectueux et adapté des enfants } \\
\text { 7.4 Force du père au plan psychologique (contrôle de soi) } \\
\text { 7.5 Capacité à bien s'organiser dans la vie quotidienne } \\
\text { 7.6 Qualité des conditions de vie et soutien de l'environnement } \\
\text { formel et informel }\end{array}$ \\
\hline $\begin{array}{l}\text { 8. Facteurs perçus comme ayant } \\
\text { contribué positivement au bon } \\
\text { fonctionnement de l'exercice du rôle } \\
\text { parental post-rupture (selon la } \\
\text { chercheure) }\end{array}$ & $\begin{array}{l}\text { 8.1 Comportement proactif du père à l'égard de ses enfants } \\
8.2 \text { Capacité à analyser de manière nuancée la dynamique familiale } \\
\text { et à focaliser sur les éléments positifs (présents et futurs) } \\
\text { 8.3 Capacité à demander de l'aide ou à l'accepter } \\
8.4 \text { Qualité des conditions de travail du père }\end{array}$ \\
\hline $\begin{array}{l}\text { 9. Difficultés et obstacles rencontrés } \\
\text { post-rupture }\end{array}$ & $\begin{array}{l}\text { 9.1 Résistance de la mère à laisser le père s'engager davantage dans } \\
\text { la vie des enfants } \\
\text { 9.2 Résistance des milieux à une présence accrûe des pères auprès de } \\
\text { leurs enfants } \\
9.3 \text { Difficultés psychologiques au plan du deuil amoureux } \\
\text { 9.4 Difficultés à assumer la paternité à temps partiel: perte du } \\
\text { contact quotidien avec les enfants } \\
\text { 9.5 Difficultés à réaménager sa vie (déménagement, changement } \\
\text { d'emploi) pour optimiser la conciliation travail-famille et vie } \\
\text { personnelle } \\
\text { 9.6 Difficultés économiques }\end{array}$ \\
\hline $\begin{array}{l}\text { 10. Conséquences positives du soutien } \\
\text { reçu (soutien formel et informel) }\end{array}$ & $\begin{array}{l}\text { 10.1 Validation et normalisation de son expérience de père séparé } \\
\text { 10.2 Meilleure canalisation des émotions négatives en vue de se } \\
\text { recentrer sur le bien-être des enfants et sur ce qui va bien } \\
\text { 10.3 Développement de stratégies d'action susceptibles de faciliter } \\
\text { l'adaptation des enfants à la rupture } \\
\text { 10.4 Plus grande affirmation de ses droits en tant que père } \\
\text { 10.5 Facilitation du processus de deuil amoureux } \\
\text { 10.6 Diminution des tracas financiers ou pour se loger } \\
\text { 10.7 Élargissement du réseau social }\end{array}$ \\
\hline
\end{tabular}




\section{LES CONDITIONS ASSOCIÉES À UNE BONNE ADAPTATION AU RÔLE PATERNEL}

\section{ANALYSE DES RÉSULTATS}

Dans la prochaine section, nous présenterons une typologie de quatre parcours paternels post-rupture (A, B, C et D) et une analyse écologique des facteurs qui sont perçus comme ayant facilité l'adaptation au rôle paternel.

\section{Les différents parcours parentaux des pères divorcés}

Les parcours parentaux renvoient aux principaux événements, obstacles, facilitateurs qui caractérisent l'expérience de vie de différents sous-groupes de pères après leur rupture conjugale.

Le parcours parental A est axé sur l'épanouissement et la continuité familliale $(n=6)$. La vie des pères de ce parcours s'est bien déroulée après la rupture, sans trop d'embûches, en garde physique partagée dès le début. ${ }^{3}$ Pour ces pères et leur ex-conjointe, cet arrangement de garde représentait la meilleure option. Ces pères étaient déjà engagés dans la vie de leurs enfants et ils pouvaient maintenir et bonifier cette implication après la rupture. Ils ne perçoivent pas la rupture comme un événement ayant exigé beaucoup d'adaptation de leur part: elle avait été planifiée, négociée et graduelle. Les principaux défis associés à leur nouveau statut conjugal-gérer seul la maison et le quotidien des enfants, redéfinir ses goûts et intérêts en tant qu'homme et en tant que père et s'entendre avec la mère de leur enfant concernant le nouveau fonctionnement familial—n'ont pas taxé leurs ressources. La principale difficulté rapportée a trait à la charge de travail durant les semaines où les enfants sont absents de la maison: ils profitaient de ce temps pour réaliser des heures supplémentaires au travail et pour terminer des tâches domestiques en vue de maximiser le temps passé avec les enfants. Ils considèrent que la séparation leur a permis de se retrouver comme individu, de se définir comme père à l'extérieur des attentes de la mère de leurs enfants et de passer du temps de meilleure qualité avec leurs enfants.

Le parcours parental B est quant à lui centré sur la protection des acquis parentaux $(n=$ 8). Les pères de ce parcours ont vécu des moments difficiles pendant et après la rupture, car elle n'avait pas été nécessairement souhaitée ou prévue. Les principales difficultés concernent le processus de séparation lui-même, surtout au plan du deuil amoureux et de la famille « unie », et au plan des quelques conflits avec l'ex-conjointe concernant l'éducation des enfants et la séparation des frais associés aux enfants. Ces difficultés ont diminué en intensité avec le temps, grâce entre autres à leurs démarches proactives pour aller se chercher du soutien de leur réseau naturel et du réseau professionnel. Ces démarches ont entre autres facilité dès les premiers moments après la rupture l'accès à des arrangements de garde satisfaisants ${ }^{4}$ et elles ont permis à ces pères de maintenir, comme avant, un engagement important dans la vie quotidienne de leurs enfants. Ces pères sont d'avis que le fait de pouvoir s'occuper de leurs enfants les ont aidés à traverser les moments difficiles à la suite de la rupture. Avec du recul, ils considèrent que même si la séparation ne représente pas un événement facile à vivre, elle leur a tout de même permis de se découvrir comme individu et comme père tout en développant des intérêts et des habiletés qu'ils n'auraient pas développés autrement.

Le parcours parental $\mathrm{C}$ est axé sur l'affirmation et la négociation des droits parentaux $(n=7)$. Pour ces pères, la transition familiale exige plusieurs réaménagements pour assurer l'accès à des conditions satisfaisantes d'exercice du rôle paternel. La vie post-rupture fut très difficile durant les 2 premières années, car ils ont vécu un important deuil amoureux et de la famille unie en plus de devoir se battre pour obtenir quelques années plus tard un arrangement de garde satisfaisant ${ }^{5}$ (une entente de garde physique partagée ou une entente 


\section{REVUE CANADIENNE DE SANTÉ MENTALE COMMUNAUTAIRE}

leur permettant de voir leurs enfants les fins de semaine, les soirs de semaine et durant les congés scolaires). Cette lutte pour la garde physique a impliqué plusieurs sacrifices et réaménagements, notamment sur le plan de la carrière, du train de vie et du lieu de résidence. Ces pères ont également vécu des conflits importants avec leur ex-conjointe relativement à l'éducation des enfants et à la séparation des biens et frais associés aux enfants. Toutefois, cette situation difficile s'est stabilisée au fil du temps grâce à des compromis et du soutien de leur environnement. Cela leur a permis de conclure une entente satisfaisante avec leur ex-conjointe concernant la garde des enfants.

Le parcours parental D est caractérisé par le maintien du rôle paternel malgré des conditions insatisfaisantes $(n=6)$. L'expérience de vie de ces pères se déroule dans un climat conjugal post-rupture qui met en péril le maintien de l'exercice du rôle paternel. Après la rupture, cette expérience fut très difficile comme dans le parcours $\mathrm{C}$, mais à la différence qu'ils n'ont pas réussi à accéder à des arrangements de garde pleinement satisfaisants (avoir accès à un droit de visite ou à une garde physique partagée). Ces pères n'ont pas réalisé autant de démarches pour modifier leurs conditions de vie personnelle et parentale que les pères du parcours $\mathrm{C}$ (principalement en raison de difficultés financières et psychologiques). Ils alimentent encore aujourd'hui face à leur ex-conjointe et au système social un sentiment assez profond d'impuissance et d'injustice. Aussi, ils adoptent un discours revanchard et politisé pour référer à leur expérience de père séparé. Certains d'entre eux sont déçus de ne pas s'être battus dès le départ pour obtenir une garde physique partagée. Malgré leurs insatisfactions par rapport à leur influence limitée dans l'éducation de leurs enfants depuis leur rupture, ils maintiennent un contact avec ceux-ci, car ils considèrent qu'il serait dommageable pour leur bien-être de les priver de leur père et ils ont l'impression qu'ils réussissent malgré tout à apporter quelque chose de bon dans leur vie. Ils espèrent que dans l'avenir l'enfant formulera le souhait de venir habiter avec eux. C'est ce qui les motive à ne pas décrocher de leur rôle parental, entre autres choses.

TABLEAU 5

Synthèse de l’intensité des difficultés rencontrées selon le parcours parental post-rupture

\begin{tabular}{|c|c|c|c|c|}
\hline Difficultés rencontrées & $\begin{array}{c}\text { Parcours } \\
\text { A }\end{array}$ & $\begin{array}{c}\text { Parcours } \\
\text { B }\end{array}$ & $\begin{array}{l}\text { Parcours } \\
\text { C }\end{array}$ & $\begin{array}{c}\text { Parcours } \\
\text { D }\end{array}$ \\
\hline $\begin{array}{l}\text { Communiquer avec l'ex-conjointe concernant } \\
\text { l'éducation des enfants }\end{array}$ & +- & + & + & ++ \\
\hline $\begin{array}{l}\text { Surmonter les sentiments dépressifs après la } \\
\text { rupture conjugale }\end{array}$ & +- & + & ++ & ++ \\
\hline $\begin{array}{l}\text { Réaménager sa vie pour favoriser la conciliation } \\
\text { travail-famille et vie personnelle }\end{array}$ & + & + & ++ & + \\
\hline S'adapter à l'absence des enfants au quotidien & +- & +- & + & ++ \\
\hline $\begin{array}{l}\text { Arriver à une entente satisfaisante avec l'ex- } \\
\text { conjointe concernant les arrangements de garde } \\
\text { physique et l'accès aux enfants }\end{array}$ & - & + & ++ & ++ \\
\hline Se redéfinir comme père & +- & +- & + & + \\
\hline Difficultés économiques & +- & +- & + & + \\
\hline S'occuper des enfants en solo & +- & +- & + & +- \\
\hline
\end{tabular}

Légende : $++=$ très difficile; $+=$ difficile; $+-=$ moyennement difficile; $-=$ aucune difficulté 


\section{LES CONDITIONS ASSOCIÉES À UNE BONNE ADAPTATION AU RÔLE PATERNEL}

Plus on se dirige vers le parcours parental D, plus les difficultés vécues sont nombreuses, plus les demandes d'adaptation sont importantes et plus le rôle paternel est mis en danger. Le tableau 5 illustre cette notion de gradation des difficultés rencontrées selon les parcours parentaux.

\section{Analyse écologique des facteurs perçus comme favorables à l'adaptation au rôle parental selon le type de trajectoire}

Les pères devaient identifier des facteurs qui, selon eux, ont contribué à leur bonne adaptation aux difficultés rencontrées. En plus de ces facteurs, notre analyse a fait ressortir aussi des éléments, qui sans être nommés directement par les pères comme « facilitants », semblent avoir joué un rôle important dans leur trajectoire parentale.

Les pères des parcours $\mathrm{C}$ et $\mathrm{D}$ considèrent que ce sont surtout leurs forces personnelles (leur capacité à faire des sacrifices et des compromis pour maintenir un lien minimal avec l'enfant) qui les ont aidés à passer au travers des difficultés rencontrées même s'ils affirment, dans la plupart des cas, avoir été bien soutenus par leur environnement. Toutefois, très peu mentionnent que leur attitude ou leur comportement pré- ou postrupture aient pu contribuer à amplifier les difficultés vécues (alors que le discours revanchard de certains a probablement envenimé les conflits entre les ex-conjoints). Cette façon globale d'analyser leur situation personnelle (réaliser de l'attribution interne concernant ses succès adaptatifs et de l'attribution externe concernant la source des problèmes rencontrés) doit protéger leur estime personnelle et jouer un rôle important concernant l'adaptation à leur situation parentale. Le tableau 6 présente une synthèse des facteurs ayant facilité l'adaptation à leur rôle paternel post-rupture. Plusieurs des caractéristiques ontosystémiques identifiées par la chercheure font écho aux travaux de Wallerstein et Kelly (1980).

Les pères des parcours $\mathrm{A}$ et $\mathrm{B}$, quant à eux, attribuent leur bonne adaptation tant à des variables de leur environnement (comportement de leur ex-conjointe, de leurs enfants et de leur employeur) qu'à leurs caractéristiques personnelles (capacité à s'être mobilisé rapidement pour négocier l'entente de garde physique). Cela peut être expliqué par le fait qu'ils se trouvent dans une situation parentale et personnelle moins précaire et menaçante au plan identitaire et personnel que les pères des parcours $C$ et D. Le tableau 7 présente une synthèse des facteurs ayant facilité l'adaptation à leur rôle paternel post-rupture.

Nous présentons dans la prochaine section une analyse plus détaillée des principaux facteurs qui semblent avoir favorisé l'adaptation des pères à leur rôle parental post-rupture.

Les facteurs ontosystémiques. Un thème très présent dans le discours des pères rencontrés (peu importe leur parcours parental) concerne l'importance qu'ils accordent à leur rôle de père et le lien fort « de sang » qui les unit à leurs enfants. Des chercheurs ont d'ailleurs observé que le maintien de l'engagement des pères divorcés est associé (a) à l'intensité de l'identification à leur rôle parental (Ihinger-Tallman et al., 1995; Le Bourdais et al., 2001; Minton \& Pasley, 1996; Stone \& McKenry, 1998; Wallerstein \& Kelly, 1980) et (b) à l'intensité du lien d'attachement aux enfants (Slater, 1999; Stone \& McKenry, 1998). Aussi, une majorité de pères rapportent que leur désir d'enfants était présent à leur esprit depuis longtemps (et non porté principalement par la mère des enfants).

Ben il y avait une motivation naturelle, un élan naturel, un lien naturel quelque chose qui est très difficile à casser, à rompre (entre moi et mes enfants), malgré les difficultés que j'ai rencontrées, mais il y avait aussi le support du groupe des pères séparés qui m'a aidé à ne pas abandonner (père 2D). 
TABLEAU 6

Synthèse des principaux facteurs perçus comme essentiels au maintien et à l'adaptation au rôle paternel post-rupture (parcours parentaux $C$ et $D$ )

\begin{tabular}{|c|c|c|}
\hline Facteurs identifiés à la suite & \multicolumn{2}{|c|}{ Facteurs directement identifiés par les pères } \\
\hline \begin{tabular}{l}
\multicolumn{1}{c}{ Facteurs ontosystémiques } \\
a) capacité de lâcher prise par \\
rapport aux domaines \\
familiaux sur lesquels ils n'ont \\
plus d'influence et d'agir sur \\
les conditions qui leur \\
permettent d'améliorer leurs \\
conditions de garde; \\
b) capacité d'analyser la \\
dynamique familiale et à \\
prendre du recul par rapport à \\
celle-ci (par exemple, ne pas \\
interpréter le comportement \\
évitant des enfants comme \\
étant dirigé contre lui, mais \\
plutôt comme étant le résultat \\
de pression subie par l'enfant); \\
c) capacité de reconnaitre les \\
impacts positifs même limités \\
qu'ils ont dans la vie de leurs \\
enfants; \\
d) capacité à être proactifs face \\
à leurs enfants; \\
e) capacité de demander ou \\
d'accepter de l'aide; \\
f) capacité à identifier les \\
effets positifs de la séparation \\
sur leur relation avec les \\
enfants.
\end{tabular} & $\begin{array}{l}\text { Facteurs ontosystémiques } \\
\text { a) capacité de faire des } \\
\text { compromis et des sacrifices pour } \\
\text { ses enfants; } \\
\text { b) avoir été impliqué dans le } \\
\text { quotidien des enfants avant la } \\
\text { rupture; } \\
\text { c) valoriser la famille; } \\
\text { d) sentir un lien affectif } \\
\text { important avec ses enfants (lien } \\
\text { de sang); } \\
\text { e) se montrer disponible pour les } \\
\text { enfants et être constant et fidèle } \\
\text { aux heures de garde convenues; } \\
\text { f) capacité à miser sur l'avenir } \\
\text { (être patient) plutôt qu'agir selon } \\
\text { des réactions émotives court- } \\
\text { terme (ne pas alimenter les } \\
\text { conflits avec l'ex-conjointe et } \\
\text { adopter une attitude de neutralité } \\
\text { dans le but de diminuer les } \\
\text { conflits d'allégeance et de } \\
\text { faciliter la relation père-enfant à } \\
\text { moyen terme); } \\
\text { g) s'être informé sur comment } \\
\text { modifier ses arrangements de } \\
\text { garde via les tribunaux ou la } \\
\text { médiation. }\end{array}$ & \begin{tabular}{l}
\multicolumn{1}{|c}{ Facteurs externes } \\
Microsystémiques et \\
mésosystémiques: \\
a) avoir des enfants qui se \\
comportent bien et qui sont \\
disciplinés; \\
b) avoir accès à des \\
ressources de soutien pour les \\
pères séparés. \\
Macrosystémiques: \\
c) se séparer dans une époque \\
où les ruptures d'union sont \\
courantes.
\end{tabular} \\
\hline
\end{tabular}

Quelques-uns précisent avoir pris davantage soin des enfants que la mère $(n=7)$. Ils appuient ces propos en précisant que ce sont eux qui donnaient les biberons, les bains, qui préparaient les repas, qui aidaient les enfants dans leurs devoirs et qui allaient les conduire chez des ami(e)s ou à l'école. D'autres affirment s'être plutôt impliqués dans la vie sportive et ludique de leurs enfants, surtout durant les fins de semaines $(n=4)$.

Ainsi, pour plusieurs pères $(n=20)$, il semble inconcevable, malgré les difficultés rencontrées, d'abandonner leurs enfants, car (a) ils les ont voulus et ils les aiment, (b) ils sont convaincus qu'ils ont un rôle à jouer dans leur développement et (c) ils se sentent responsables de leur assurer un bon avenir. 
TABLEAU 7

Synthèse des principaux facteurs perçus comme essentiels au maintien et à l'adaptation au rôle paternel post-rupture (parcours parentaux $A$ et B)

\begin{tabular}{|c|c|c|}
\hline & \multicolumn{2}{|c|}{ Facteurs directement identifiés par les pères } \\
\hline $\begin{array}{l}\text { Facteurs ontosystémiques } \\
\text { a) avoir de bons revenus; } \\
\text { b) avoir le souci d'assurer une } \\
\text { continuité familiale dans la vie } \\
\text { des enfants et de les soutenir } \\
\text { dans leur adaptation à la } \\
\text { rupture; } \\
\text { c) capacité à être proactifs face } \\
\text { à leurs enfants: leur témoigner } \\
\text { concrètement qu'ils les aiment, } \\
\text { aller au devant d'eux en leur } \\
\text { téléphonant, en se renseignant } \\
\text { sur leur vie et en trouvant de } \\
\text { nouvelles activités plaisantes à } \\
\text { faire avec eux; } \\
\text { d) capacité de demander ou } \\
\text { d'accepter de l'aide; } \\
\text { e) concevoir que l'ex-conjointe } \\
\text { est une bonne mère pour ses } \\
\text { enfants et que son rôle s'inscrit } \\
\text { en complémentarité avec le } \\
\text { leur. }\end{array}$ & \begin{tabular}{l}
\multicolumn{1}{c}{ Facteurs ontosystémiques } \\
a) capacité de faire des \\
compromis et des sacrifices pour \\
ses enfants; \\
b) avoir été impliqué dans le \\
quotidien des enfants avant la \\
rupture; \\
c) s'être informé rapidement \\
pour savoir comment négocier \\
l'entente de séparation et les \\
arrangements de garde; \\
d) valoriser la famille; \\
e) sentir un lien affectif \\
important avec ses enfants (lien \\
de sang); \\
f) disposer de temps pour soi et \\
pour préparer la semaine de \\
garde quand les enfants ne sont \\
pas là; \\
g) impliquer les enfants dans la \\
gestion des tâches quotidiennes; \\
h) détenir un emploi qui facilite \\
la conciliation travail-famille.
\end{tabular} & \begin{tabular}{l}
\multicolumn{1}{c}{ Facteurs externes } \\
Microsystémiques: \\
a) ne pas avoir rencontré de \\
résistance importante de la \\
part de la mère concernant la \\
garde physique et avoir \\
officialisé l'entente de garde \\
physique; \\
b) avoir discuté et préparé la \\
rupture avec l'ex-conjointe; \\
c) avoir des enfants qui \\
s'adaptent bien à la rupture et \\
au nouveau contexte de garde. \\
Exosystémique: \\
a) avoir accès à un service \\
gratuit de médiation familiale.
\end{tabular} \\
\hline
\end{tabular}

Plusieurs mentionnent que la séparation représente un événement coûteux et que la garde physique partagée exige un revenu minimal pour assurer le bien-être des enfants au sein de deux maisons ou appartements $(n=19)$. D'ailleurs, la plupart des pères qui détiennent la garde physique partagée bénéficient de revenus plus élevés et d'horaires de travail plus souples $(80 \%$ des pères avec une garde partagée disposaient d'un revenu annuel brut supérieur à $40000 \$$ comparativement à $40 \%$ pour ceux qui ne participent pas à cette modalité de garde). Certains mentionnent que le fait d'avoir été très impliqués dans la gestion de la vie domestique avant la séparation les aide à bien s'organiser au quotidien.

Enfin, une autre caractéristique ontosystémique qui ressort particulièrement dans cette étude réfère à la capacité des pères à avoir demandé du soutien (et pas seulement celui en provenance du réseau informel) ou à l'avoir accepté lorsque suggéré. Même si certains des participants réfèrent au rapport difficile qu'entretiennent les hommes à la demande d'aide et au fait que peu de ressources sont spécialement dédiées à soutenir les pères, la majorité d'entre eux ont accepté ou cherché le soutien de l'environnement formel: $58 \%$ des pères avaient utilisé les services d'un médiateur familial; $56 \%$ avaient demandé de l'aide à un psychologue (typiquement du programme d'aide aux employés) ou à un travailleur social ou travailleuse sociale (du CLSC) pour les soutenir dans leur processus de séparation et de gestion de leur rôle parental. De plus, $28 \%$ des participants ont fait partie d'un groupe de 


\section{REVUE CANADIENNE DE SANTÉ MENTALE COMMUNAUTAIRE}

soutien pour pères séparés, d'un groupe de soutien pour les familles monoparentales et recomposées ou du réseau Hommes Québec.

Les facteurs microsystémiques et mésosystémiques. Après la séparation, en l'absence du soutien émotionnel de l'ex-conjointe, qui représente la principale forme de soutien pour les hommes en union conjugale (Douherty, Kouneski, \& Erickson, 1998), le réseau informel devient très important pour les pères. Chez les pères rencontrés, le soutien familial prend une importance particulière, surtout durant la première année qui a suivi la rupture: les parents ont offert un hébergement temporaire et les ont soutenus dans leur rôle parental (surtout concernant les pères des parcours $\mathrm{B}, \mathrm{C}$ et $\mathrm{D}$ ). Ce soutien a été très apprécié, car plusieurs rapportent avoir vécu des épisodes dépressifs importants et des idéations suicidaires.

Les enfants, à leur façon, jouent également un rôle dans le maintien de l'engagement des pères, notamment lorsqu'ils témoignent au père leur intérêt à venir chez lui. Dans certains cas, ce sont même les enfants qui motivent les pères à revendiquer pour modifier leurs arrangements de garde.

Au bout de 4 ans mon petit bonhomme m'a demandé [. . .]: « Est-ce que je pourrais venir faire des dodos chez toi papa est-ce que c'est correct? »C'est de là que j'ai commencé à entreprendre là [les démarches] pour une garde partagée (père 24C).

Enfin, les ami(e)s et les collègues de travail offrent un soutien moral et psychologique aux pères durant les moments où ils sont à hauts risques d'abandonner leur rôle parental. Ce soutien leur permet de prendre du recul par rapport aux conflits et aux insatisfactions vécues, les aidant ainsi à analyser leur situation familiale dans une autre perspective et à se recentrer sur le bien-être de leur enfant.

\section{DISCUSSION}

Pourquoi les pères rencontrés maintiennent-ils leur engagement auprès de leurs enfants, même si dans certains cas, ils rencontrent des difficultés importantes?

La plupart des difficultés rapportées par les pères dans le cadre de cette étude sont similaires à celles identifiées par d'autres chercheurs qui ont voulu comprendre la réalité des pères divorcés (Gaudet et al., 2005). Il semble que la perte du contact avec les enfants au quotidien de même que les conflits avec l'ex-conjointe constituent les principales difficultés vécues par les pères après une rupture conjugale. Là où notre étude se distingue et permet de scruter de manière différente et complémentaire la paternité post-divorce tient au fait qu'elle vise à comprendre non pas les processus de déliaison père-enfant ou de décrochage paternel, mais plutôt à identifier les contextes de vie qui sont favorables au maintien du contact père-enfant, même si dans certaines situations ce contact était minimal.

Chez les pères des parcours $\mathrm{A}$ et $\mathrm{B}$, la question du décrochage complet de leur rôle parental ne se pose pas: ils veulent être impliqués dans le quotidien de leurs enfants et ils disposent de modalités de garde satisfaisantes pour y arriver. Aussi, la grande implication paternelle pré-rupture rapportée par la majorité d'entre eux semble les avoir aidés à accéder à de bonnes conditions d'exercice de leur rôle parental. En effet, ils avaient, selon eux, déjà fait « leurs preuves» en tant que pères au quotidien, donc l'exercice de leur rôle paternel à temps partagé n'était pas remis en question par la mère. Bien que la séparation ait engendré son lot d'insécurités et de souffrances, elle leur a permis de s'épanouir comme individus. De plus, elle leur a permis de développer un mode de fonctionnement paternel plus satisfaisant, à l'extérieur des attentes quotidiennes de la mère de leurs enfants et de la 
routine de vie familiale plus ou moins satisfaisante qui s'était installée malgré eux au fil des années. À cet effet, certains chercheurs montrent que cette capacité à considérer une perte ou un événement difficile (telle que la rupture conjugale) comme un événement salutaire sur le plan du développement personnel constitue une stratégie d'adaptation efficace qui protège l'estime de soi (Davis, Nolen-Hoeksema, \& Larson, 1998; Murray, 2001).

Toutefois, chez les pères des parcours parentaux $\mathrm{C}$ et $\mathrm{D}$, il y a peu d'éléments associés à une bonne adaptation aux transitions familiales (Cloutier et al., 1997). En effet, ces pères n'ont pu planifier leur rupture, car dans la plupart des cas (9 pères sur 13) elle leur avait été imposée. Aussi, ils disposent de relativement peu de pouvoir (réel ou perçu) sur leurs arrangements de garde ou l'éducation de leurs enfants. Plusieurs vivent dans des conditions économiques précaires ( 7 des 9 pères qui gagnent entre $20000 \$$ et $39999 \$$ par année sont de type $\mathrm{C}$ ou $\mathrm{D}$ ) et ils ne peuvent maintenir le même niveau d'engagement parental qu'auparavant. Cependant, certains facteurs de protection semblent avoir limité l'effet négatif de ces obstacles sur l'adaptation à leur rôle paternel post-rupture.

En effet, la majorité valorisent leur rôle de père, et certains lui accordent autant d'importance sinon plus qu'à leur rôle d'employé, d'ami ou d'amoureux. Les pères du parcours $\mathrm{C}$ ont donc réalisé plusieurs démarches en vue de modifier leurs modalités de garde physique. Comme ces démarches ont porté fruits, cela leur permet d'actualiser pleinement leur valeur familiale et de maintenir une identité paternelle congruente à leurs aspirations. Pour eux, cette modalité de garde devenait un élément incontournable au maintien de leur engagement. Cela rejoint d'ailleurs les constats de Kruk (1991) et de Lewis et al. (1997): les pères qui étaient très engagés dans la vie de leurs enfants avant la rupture et qui ne peuvent maintenir un tel niveau d'engagement après sont plus à risque de cesser de voir leurs enfants que ceux qui étaient modérément engagés dans leur quotidien.

En ce qui concerne les pères du parcours D, cette valeur familiale les incite à ne pas décrocher complètement de leur rôle parental. Toutefois, comme elle ne peut s'actualiser comme ils le souhaiteraient, ils doivent recourir plus intensément à des stratégies d'adaptation cognitives très spécifiques: se centrer sur les quelques éléments positifs de leur situaion (qualité des moments passés avec leurs enfants) et croire en un avenir meilleur avec leurs enfants.

Enfin, le fait que plusieurs des pères de ces deux parcours acceptent ou demandent du soutien de la part de leur réseau social formel et informel semble contribuer à diminuer l'intensité des difficultés rencontrées. Cette étude fait ressortir l'importance de la proximité des ressources de soutien pour les pères séparés: la majorité ont consulté le psychologue du milieu du travail ou des ami(e)s et la famille.

\section{CONCLUSION}

Cette recherche ne vise pas à dresser un portrait représentatif de l'ensemble des pères séparés du Québec: la majorité d'entre eux ne sont pas aussi engagés que ceux que nous avons rencontrés. Cependant, en dépit du fait que cette étude s'est réalisée auprès d'un petit échantillon de pères, la saturation des analyses et la procédure de validation auprès des participants nous indiquent qu'elle représente bien la réalité d'un sous-groupe de pères peu documentée, soit ceux qui sont motivés à demeurer présents dans la vie de leurs enfants après une rupture. De plus, elle se situe en toute complémentarité avec les travaux des chercheurs canadiens dans le domaine. 


\title{
REVUE CANADIENNE DE SANTÉ MENTALE COMMUNAUTAIRE
}

Cette analyse témoigne d'un changement de croyances et de pratiques relativement à la place du père pour ses enfants. Il s'agit toutefois d'un changement perçu comme étant localisé à certaines familles, puisque les pères des parcours $\mathrm{A}$ et $\mathrm{B}$ se sentent privilégiés, par rapport à d'autres, de pouvoir avoir accès aussi facilement à leurs enfants. L'image sociale négative du «père McDo de fin de semaine » semble encore bien présente à l'esprit des pères que nous avons rencontrés: certains veulent s'en dissocier même s'ils jouent essentiellement un rôle ludique auprès de leurs enfants, tandis que d'autres la condamnent et la jugent sévèrement.

Ainsi, selon les pères rencontrés, il ne semble pas que ce soit le stigma social d'être un homme divorcé qui pèse lourd dans les années 2000: les ruptures sont de plus en plus fréquentes. Il s'agit plutôt du fait d'être, dans certains cas, un père qui valorise sa paternité et qui est mis à l'écart du noyau mère-enfants, surtout dans un contexte social où l'engagement paternel est de plus en plus valorisé. Il semble donc que la garde physique partagée doit être encouragée lorsque les parents ont montré dans le passé et continuent d'afficher après la rupture une motivation et une réelle capacité à exercer leur rôle parental (comme cela semblait être le cas chez la plupart des pères rencontrés).

Toutefois, si on aspire à ce qu'un plus grand nombre de familles puissent disposer d'un tel arrangement de garde, il faut en contrepartie leur donner accès à du soutien financier, psychologique et social (notamment par le biais de conditions de travail favorables à la conciliation travail-famille), et ce de façon rapide et intensive durant les premières années qui suivent la rupture.

\section{NOTES}

1. Ce nombre de participants est justifié par le fait que nous avons voulu rencontrer tous les pères qui se montraient intéressés à participer à l'étude, même si nos analyses ont atteint un niveau de saturation intéressant après les 23 premières entrevues.

2. Ce critère est justifié par le fait que plusieurs études montrent que le lien père-enfant tend à se fragiliser 2 à 5 années après la séparation (Marcil-Gratton \& Le Bourdais, 1999; Wallerstein \& Kelly, 1980).

3. Lorsqu'un parent habite avec son enfant au moins $40 \%$ du temps, la garde physique est partagée.

4. Seulement un de ces pères n'avait pas officiellement un arrangement de garde physique partagée, mais il pouvait voir son enfant durant les soirs de semaine en plus de ses droits de visite durant les fins de semaine et les congés scolaires.

5. Deux de ces pères n'avaient pas obtenu une garde physique partagée.

\begin{abstract}
This article presents the findings of one phase of a qualitative study aimed at identifying the various factors (psychological and social) that seem to help or hinder fathers in coping with their parental role after a marital breakdown. Analyses of the life stories of 27 separated or divorced fathers who are present in their children's lives identified 4 paternal coping trajectories following marital breakdown. These trajectories or life paths refer to the events, obstacles, strategies, and facilitating factors that characterize the fathers' experiences after separation or divorce. The factors that have a positive influence on how they cope with their parental role vary according to trajectory. However, a number are common to the group of fathers as a whole. These concern a father's ability to make significant compromises and sacrifices with his expartner in relation to their separation or divorce agreement, his belief in the importance of the role he plays in his children's lives, his ability to accept or ask for help after the
\end{abstract}




\section{LES CONDITIONS ASSOCIÉES À UNE BONNE ADAPTATION AU RÔLE PATERNEL}

breakdown of his marriage and his proactive approach to achieving satisfactory conditions for carrying out his parental role.

\section{RÉFÉRENCES}

Allard, F.L., Bourret, A., Tremblay, G., et coll. (2004). Rester engagé envers son enfant après la rupture du couple: Point de vue de pères vivant en contexte de pauvreté. Beauport, QC: Agence de développement de réseaux locaux de services de santé et de services sociaux de la Capitale nationale, Direction de la santé publique.

Arditti, J.A., \& Bickley, P. (1996). Fathers' involvement and mothers' parenting stress postdivorce. Journal of Divorce and Remarriage, 26(1-2), 1-23.

Bronfenbrenner, U. (1979). The ecology of human development: Experiments in nature and by design. Cambridge, MA: Harvard University Press.

Cloutier, R., Beaudry, M., Drapeau, S., Samson, C., Mireault, G., Simard, M., et al. (1997). Changements familiaux et continuité: une approche théorique de l'adaptation aux transformations familiales. Dans G.M. Tarabulsy \& R. Tessier (dir.), Enfance et famille: contextes et développement (pp. 28-56). Québec, QC: Presses de l’Université du Québec.

Comité de travail en matière de prévention et d'aide aux hommes. (2004). Les hommes: s'ouvrir à leur réalité et répondre à leurs besoins. Québec, QC: Ministère de la Santé et des Services sociaux du Québec.

Cooksey, E.C, \& Craig, P.H. (1998). Parenting from a distance: The effects of paternal characteristics on contact between nonresident fathers and their children. Demography, 35(2), 187-201.

Davis, C.G., Nolen-Hoeksema, S., \& Larson, J. (1998). Making sense of loss and benefiting from the experience: Two construals of meaning. Journal of Personality and Social Psychology, 75(2), 561-574.

Douherty, W.J., Kouneski, E.F., \& Erickson, M.F. (1998). Responsible fathering: An overview and conceptual framework. Journal of Marriage and the Family, 60, 277-292.

Gaudet, J., Devault, A., \& Bouchard, C. (2005). Le maintien de l'exercice du rôle paternel après une rupture conjugale: obstacles et facilitateurs. Revue de Psycho-Éducation, 34(1), 21-40.

Glasser, B., \& Strauss, A. (1967). The discovery of grounded theory. Chicago: Aldine.

Hetherington, M.E., \& Kelly, J. (2002). For better or for worse: Divorce reconsidered. New York: W.W. Norton.

Ihinger-Tallman, M., Pasley, K., \& Buehler, C. (1995). Developing a middle-range theory of father involvement postdivorce. Dans W. Marsiglio (dir.), Fatherhood: Contemporary theory, research, and social policy (pp. 57-77). Thousand Oaks, CA: Sage.

Kruk, E. (1991). Discontinuity between pre- and post-divorce father-child relationships: New evidence regarding paternal disengagement. Journal of Divorce and Remarriage, 16, 195-227.

Le Bourdais, C., Juby, H., \& Marcil-Gratton, N. (2001). Maintien des contacts pères/enfants après la séparation: le point de vue des pères (CSR-2000-3E/3F ed.). Ottawa: Ministère de la Justice du Canada.

Lewis, C., Maka, Z., \& Papacosta, A. (1997). Why do fathers become disengaged from their children's lives?: Maternal and paternal accounts of divorce in Greece. Journal of Divorce and Remarriage, 28(1-2), 89-117.

Marcil-Gratton, N., \& Le Bourdais, C. (1999). Garde des enfants, droits de visite et pension alimentaire: résultats tirés de l'Enquête longitudinale sur les enfants et les jeunes. Montréal: Université de Montréal, Centre interuniversitaire d'études démographiques.

Minton, C., \& Pasley, K. (1996). Fathers' parenting role identity and father involvement. Journal of Family Issues, 17(1), 26-45.

Murray, J.A. (2001). Loss as a universal concept: A review of the literature to identify common aspects of loss in diverse situations. Journal of Loss and Trauma, 6, 219-241.

Ottosen, M.H. (2001). Legal and social ties between children and cohabiting fathers. Childhood: A Global Journal of Child Research, 8(1), 75-94.

Paillé, P. (1994). L'analyse par théorisation ancrée. Cahiers de Recherche Sociologique, 23, 244-260. Patton, M.Q. (1987). How to use qualitative methods in evaluation. Thousand Oaks, CA: Sage. 


\section{REVUE CANADIENNE DE SANTÉ MENTALE COMMUNAUTAIRE}

Rettig, K.D., Leichtentritt, R.D., \& Stanton, L.M. (1999). Understanding noncustodial fathers' family and life satisfaction from resource theory perspective. Journal of Family Issues, 20(4), 507-538.

Rosenbaum, W.L. (2000). Variables associated with the involvement and frequency of contact of nonresidential fathers with their children following divorce. Thèse de doctorat non publiée, University of New Orleans, New Orleans, LA.

Rutter, M. (1987). Psychosocial resilience and protective mechanisms. American Journal of Orthopsychiatry, 57, 316-331.

Slater, L.B. (1999). Attachment and paternal investment in divorced fathers. Thèse de doctorat non publiée, University of Washington, Seattle.

Stephens, L.S. (1996). Will Johnny see Daddy this week?: An empirical test of three theorical perspectives of postdivorce contact. Journal of Family Issues, 17(4), 466-489.

Stone, G. (2001). Father postdivorce well-being: An exploratory model. Journal of Genetic Psychology, 162(4), 460-467.

Stone, G., \& McKenry, P. (1998). Nonresidential father involvement: A test of mid-range theory. Journal of Genetic Psychology, 159(3), 313-336.

Strauss, A., \& Corbin, J. (1998). Basics of qualitative research: Techniques and procedures for developing Grounded Theory. Thousand Oaks, CA: Sage.

Wallerstein, J.S., \& Kelly, J.B. (1980). Surviving the breakup: How children and parents cope with divorce. New York: Basic Books. 